\title{
Programmable shunt valve interactions with osseointegrated hearing devices
}

\author{
Matthew J. Pierson, MD, ${ }^{1}$ Daniel Wehrmann, MD, ${ }^{2}$ J. Andrew Albers, PhD, ${ }^{1}$ \\ Najib E. El Tecle, MD, MS, ${ }^{1}$ Dary Costa, MD, ${ }^{2}$ and Samer K. Elbabaa, MD' ${ }^{1}$ \\ 1Division of Pediatric Neurosurgery, Department of Neurological Surgery; and 2Division of Pediatric Otolaryngology/ENT, \\ Department of Otolaryngology-Head and Neck Surgery, Saint Louis University School of Medicine, St. Louis, Missouri
}

\begin{abstract}
OBJECTIVE Patients with ventriculoperitoneal (VP) shunts with programmable valves who would benefit from osseointegrated hearing devices (OIHDs) represent a unique population. The aim of this study was to evaluate the magnetic field strengths of 4 OIHDs and their interactions with 5 programmable VP shunt valves.

METHODS Magnetic field strength was measured as a function of distance for each hearing device (Cochlear Baha 5, Cochlear Baha BP110, Oticon Ponto Plus Power, and Medtronic Sophono) in the following modes: inactive, active in quiet, and active in 60 decibels of background noise in the sound booth. The hearing devices were introduced to each shunt valve (Aesculap proGAV, Aesculap proGAV 2.0, Codman Hakim, Codman Certas, and Medtronic Strata II) also as a function of distance in these identical 3 settings. Each trial was repeated 5 times. Between each trial, the valves were assessed for a change in setting. Finally, using a skull model, the devices were introduced to each other in standard anatomical locations and the valves were assessed for a change in settings.
\end{abstract}

RESULTS The maximum magnetic field strengths generated by the Cochlear Baha 5, BP110, and Oticon OIHDs were $1.1,36.2$, and 48.7 gauss $(\mathrm{G})$, respectively. The maximum strength generated by the Sophono device was $>800 \mathrm{G}$. The magnetic field strength of the hearing devices decreased markedly with increasing distance from the device. The strength of the Sophono's magnetic attachment decreased to $34.8 \mathrm{G}$ at $5 \mathrm{~mm}$. The Codman Hakim, Codman Certas, and Medtronic Strata II valve settings changed when rotating the valves next to the Sophono abutment. No other changes in valve settings occurred in the distance or anatomical models for any other trials.

CONCLUSIONS This is the first study evaluating the interaction between OIHDs and programmable VP shunt valves. The findings suggest that it is safe to use these devices together without having to switch to a nonprogrammable valve or move the shunt valve to a more distant location. Still, care should be taken if the Sophono device is used to ensure that the valve is $\geq 5 \mathrm{~mm}$ away from the magnetic attachment.

https://thejns.org/doi/abs/10.3171/2016.11.PEDS16501

KEY WORDS programmable shunt valve; osseointegrated hearing device, magnetic field strength; surgical technique; hydrocephalus

$\mathrm{H}$ YDROCEPHALUS is a common neurosurgical condition necessitating CSF diversion, most commonly in the form of ventriculoperitoneal (VP) shunt placement. Classically, shunt valves had various fixed methods of regulating CSF drainage. When a patient would require a different setting than that of their current valve, they required a valve exchange operation. Programmable shunt valves have come into favor as a means to allow multiple shunt valve settings without requiring additional surgery. Programmable shunt valves have become increasingly commonplace; they allow for easy adjustment of the drainage rate of shunt systems via external, typically magnet-based, devices. The ability to adjust valve function could lead to fewer revisions when compared with nonprogrammable valves, and therefore may provide a cost benefit. ${ }^{4}$ The ease of adjustment of these valves via a magnetic field comes with the downside of erroneous valve adjustment when exposed to other magnetic fields. This has been investigated in the setting of magnetic toys, magnetic tablet covers, cell phones, televisions, amusement park rides, and head phones..$^{2,5-9,11}$ Factors that have been implicated in the effect of magnetic fields on programmable shunt valves in- 
clude distance to valve, field strength, and rotational component of the magnetic field in relation to the valve.

Patients with hearing loss and a VP shunt represent a unique population. Data on the interaction between hearing devices and programmable shunt valves are limited. Previous studies focus primarily on the interaction between cochlear implants (CIs) and programmable shunt valves. ${ }^{1,10}$ The osseointegrated hearing device (OIHD) is a rehabilitative device indicated for patients with conductive hearing loss or single-sided deafness. These devices transmit sound energy to the cochlea by vibrating the skull via a snug headband, an abutment screw, or transcutaneously to a magnet attached to the skull. To our knowledge, there are no data regarding the interaction between OIHDs and programmable shunt valves. This study is an in vitro investigation of the magnetic fields produced by OIHDs and their interaction with programmable shunt valves.

\section{Methods}

This was an in vitro study to examine the magnetic field emitted by 4 OIHDs (Cochlear Baha 5, Cochlear Baha BP 110, Oticon Ponto Plus Power, and Medtronic Sophono A2) and their interactions with 5 programmable shunt valves: Medtronic Strata II, Codman Certas, Codman Hakim, Aesculap proGAV, and Aesculap proGAV 2.0 valves. All measurements were collected in a sound booth. Magnetic field strength measurements were obtained using a DC Gaussmeter (model GM-1-HS; AlphaLab, Inc.).

The interactions between the hearing devices and valves were evaluated using distance modeling and anatomical modeling. For anatomical modeling, a full-sized adult skull was modeled in 3D by using InVesalius (CTI) to isolate bone density from a CT image of an anonymized 17-year-old boy. The rendered model was then loaded into a $3 \mathrm{D}$ slicing engine for partitioning into $0.2-\mathrm{mm}$ layers, which were then printed using acrylonitrile butadiene styrene plastic deposited by a MakerBot Replicator 3D printer (Fig. 1). The cranial model was assessed for accuracy with measurements of anteroposterior length, lateral width, and circumference. There was no tissue layer placed over the model skull to mimic the soft tissues of the scalp.

\section{Measurements of Magnetic Field Strength Generated by OIHDs}

A measuring tape was secured to a flat, nonferrous surface with the Gaussmeter at the 0 -mm mark. The magnetic field emitted by each OIHD was recorded with each inactivated device $0 \mathrm{~mm}$ from the Gaussmeter and at successive $5-\mathrm{mm}$ increments, to a distance of $50 \mathrm{~mm}$. Five readings were taken at each distance increment and the mean value was calculated. In all trials, whether with the hearing devices, Gaussmeter, or shunt valves, the 2 devices being analyzed were held at the measured distance for several seconds. The procedure was repeated with each OIHD activated at the highest volume setting in quiet. The procedure was then repeated with each device activated in 60 decibels $(\mathrm{dB})$ of speech noise generated by a GSI 61 audiometer (Grason Stadler). For each device, the maximum magnetic field strengths in the inactive, active, and active with background noise states were compared using

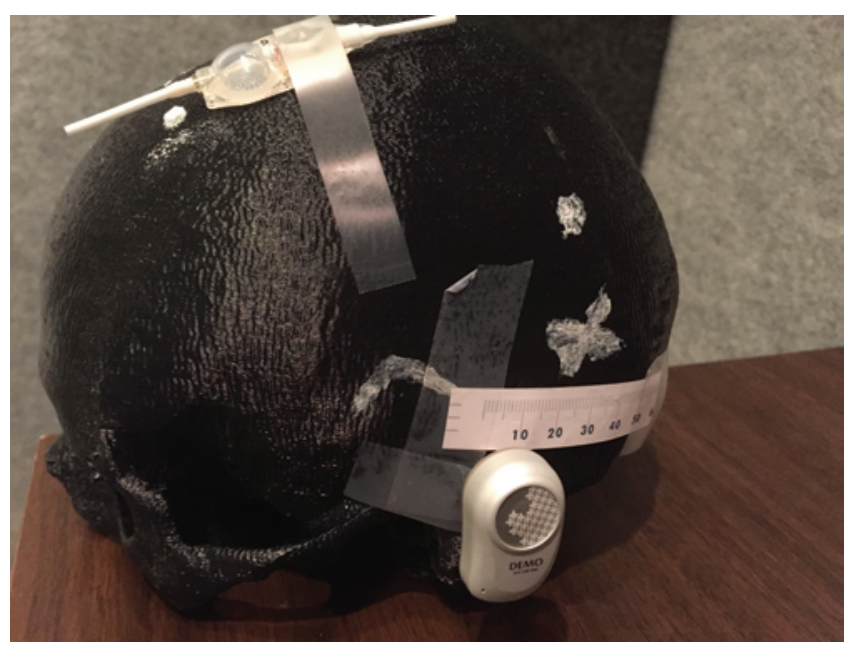

FIG. 1. Skull model with an OIHD in the standard anatomical position, with a programmable shunt valve in the ipsilateral frontal location. Note the parietooccipital " $X$ " mark used for the ipsilateral parietooccipital valve location. Figure is available in color online only.

a 1-way ANOVA, with Bonferroni correction for post hoc analysis.

\section{Evaluating the Interaction Between OIHDs and Programmable Shunt Valves via a Distance Model}

A 6-mm Oticon abutment screw was secured into the standard anatomical position on the skull model, $5.5 \mathrm{~cm}$ posterior and superior to the external auditory canal. A 50$\mathrm{mm}$ measuring tape was placed adjacent to the abutment. Each hearing device was then sequentially placed onto the abutment. The Medtronic Sophono hearing device was secured to the skull model in the anatomical location via the provided headband. The programmable valves were set to a middle setting (to allow for potential reprogramming above or below the starting point) and confirmed with each valve's respective programmer. Each valve was placed adjacent to the abutment coupled with the hearing device, with the device in inactive, active in quiet, and active in $60 \mathrm{~dB}$ of noise (to approximate speech) mode. For each hearing device environment, the shunt valve setting was measured at $0 \mathrm{~mm}$ with a rotational component to the hearing device, $0 \mathrm{~mm}$ without a rotational component, and at 5-mm increments again to $50 \mathrm{~mm}$ of total distance from the hearing device or abutment. The rotational component was tested by placing the shunt valve in direct contact with the hearing device and turning it $360^{\circ}$, followed by checking the setting. Each variable was tested 5 times, with the valve setting confirmed between each trial. In the case of the Codman Hakim valve, the setting of the device after programming was confirmed with visual inspection of the valve.

\section{Evaluating the Interaction Between OIHDs and Programmable Shunt Valves via an Anatomical Model}

The abutment in standard anatomical position for OIHD placement mentioned above was used, as well as the headband for the Medtronic Sophono device. With the hearing devices secured in the appropriate position, they were activated with the highest volume setting in $60 \mathrm{~dB}$ of noise. Four standard shunt valve locations were chosen on the 


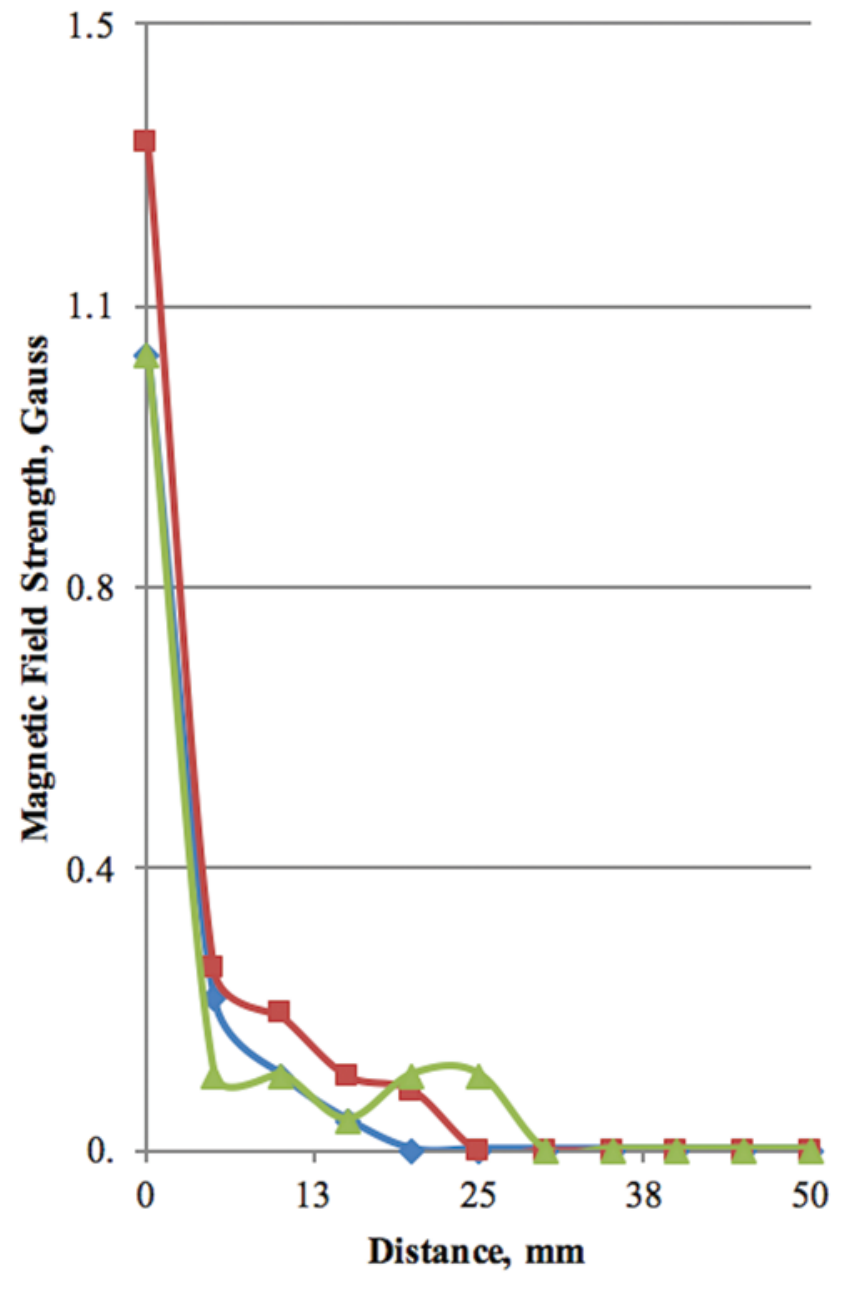

$\neg$ Inactive $\quad-$ Active $\quad-$ Active + Noise

FIG. 2. Graph of magnetic field strength as a function of distance from the Cochlear Baha 5. Figure is available in color online only.

skull model to assess for interactions. The locations were as follows: ipsilateral parietooccipital, ipsilateral frontal, contralateral parietooccipital, and contralateral frontal. The parietooccipital bur hole sites were marked using an approximation of Keen's point, with the valve seated $3 \mathrm{~cm}$ distal to the bur hole. Variations in surgeon technique may place the valve slightly closer or further away in any of the positions. The frontal bur holes were selected using Kocher's point, with the valve seated $3 \mathrm{~cm}$ distal to the bur hole. With the valves secured to the skull, each hearing device was introduced to its appropriate attachment device (abutment or magnet) to simulate a patient attaching his or her hearing device. This was repeated 5 times for each hearing device, with each shunt valve at each anatomical location and with the shunt valve setting examined after each trial.

\section{Results}

\section{Magnetic Field Strength}

Each OIHD generated a measurable magnetic field. There were no significant differences in maximum mag-

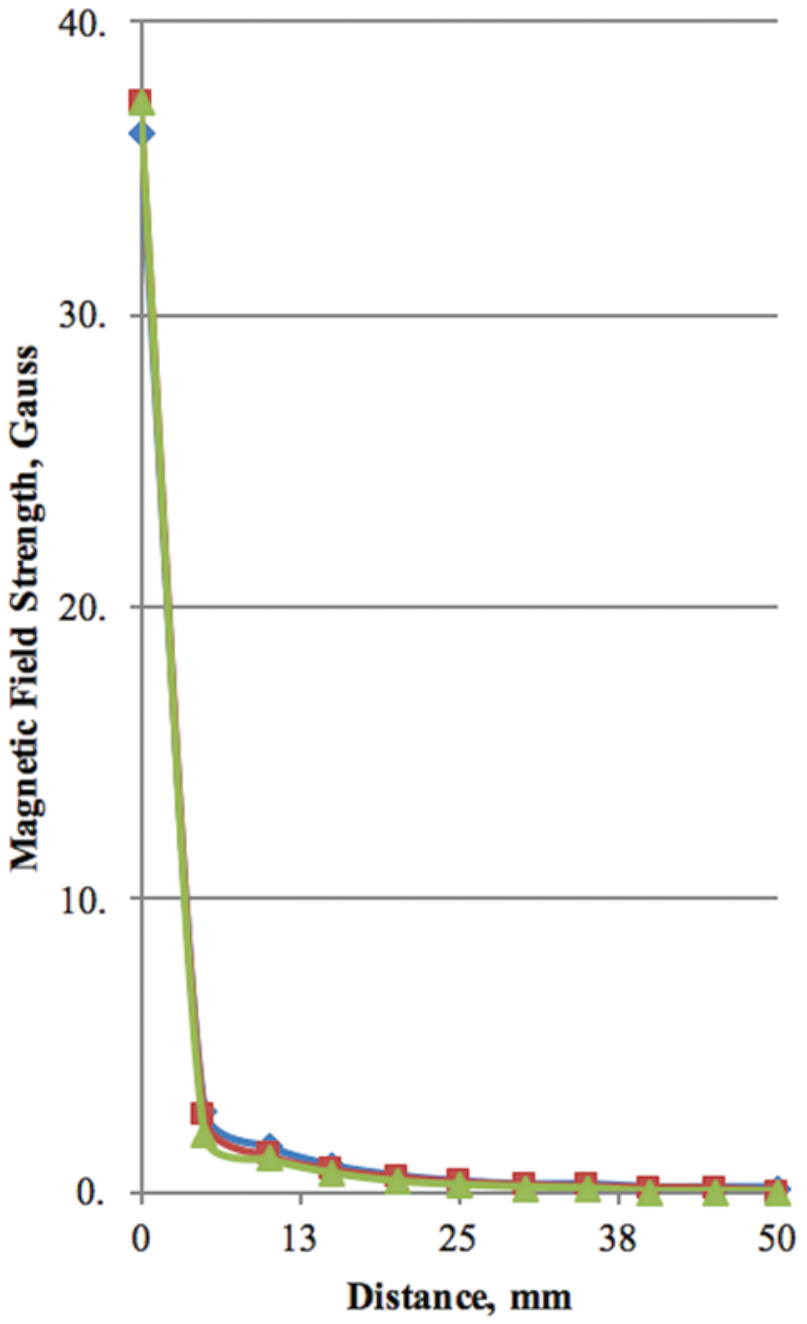

\section{$\longrightarrow$ Inactive $\quad-$ Active $\longrightarrow$ Active + Noise}

FIG. 3. Graph of magnetic field strength as a function of distance from the Cochlear BP110. Figure is available in color online only.

netic field strength generated by the device in the inactive, active, or active in background noise state for the Cochlear Baha 5, Cochlear BP110, or Sophono OIHD. However, a 1-way ANOVA was significant for differences in the maximum magnetic field generated by the Oticon Ponto Plus Power OIHD in the inactive, active, and active in background noise states $(\mathrm{F}(2,12)=171.5, \mathrm{p}<0.001)$. Post hoc analysis with Bonferroni correction revealed that the mean magnetic field generated by the Ponto Plus Power in the inactive state $(48.7 \pm 7.4 \mathrm{G})$ was significantly greater than the mean in either the active state $(3.3 \pm 0.4 \mathrm{G}, \mathrm{p}<$ $0.001)$ or active with background noise state $(7.3 \pm 0.2 \mathrm{G}$, $\mathrm{p}<0.001)$. The magnetic field values are expressed as the mean \pm SD throughout. The magnetic field strengths as a function of distance for each device in the inactive, active, and active in noise states are illustrated in Figs. 2-6, and a comparison of the maximal field strengths generated by each device in the inactive, active, and active in noise states is illustrated in Fig. 7.

The maximum magnetic fields generated by each de- 


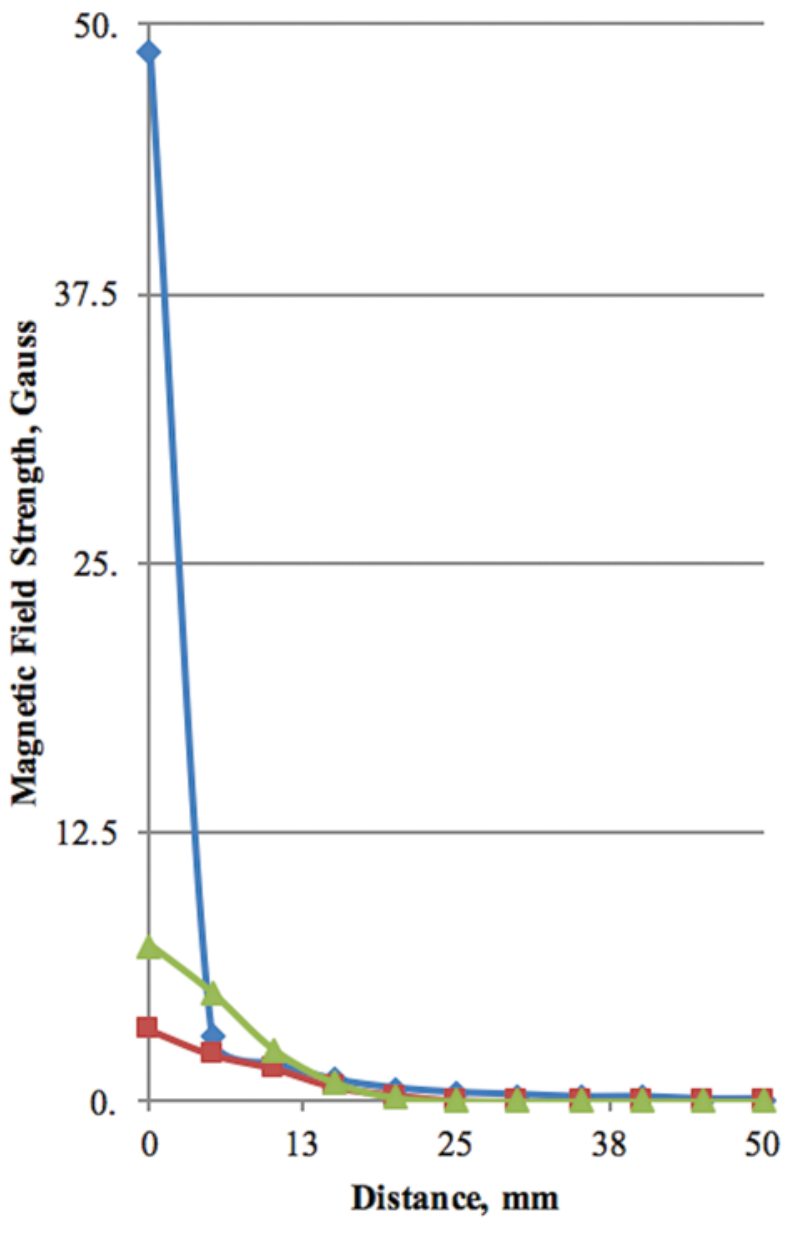

$\checkmark$ Inactive $\quad-$ Active $\quad-$ Active + Noise

FIG. 4. Graph of magnetic field strength as a function of distance from the Oticon Ponto Plus Power. Figure is available in color online only.

vice in the inactive, active, and active in noise states were compared using a 1-way ANOVA with Bonferroni correction. The ANOVA results were significant for differences between devices in the inactive $(\mathrm{F}(4,20)=5.11 \mathrm{E} 5, \mathrm{p}$ $<0.001)$; active $(\mathrm{F}(4,20)=8.22 \mathrm{E} 5.1, \mathrm{p}<0.001)$; and active in noise $(F(4,20)=3.86 E 6, p<0.001)$ states. The Sophono OIHD with magnetic abutment generated a large magnetic field that was above the limit of detection of the Gaussmeter, which was an order of magnitude greater than all other devices. When excluding the Sophono with magnetic abutment, the BP110 device generated the strongest magnetic field in the active (mean $37.2 \pm 1.7 \mathrm{G}$ ) and active in noise $(37.3 \pm 0.9 \mathrm{G})$ states, whereas the Oticon Ponto Plus Power generated the largest magnetic field in the inactive state (mean $48.7 \pm 7.4 \mathrm{G}$ ). A summary of the mean differences in magnetic field strength between devices is found in Table 1.

The maximum magnetic fields generated were $>800$ G (Sophono with abutment); 48.7 G (Oticon Ponto Plus Power); 36.2 G (Cochlear BP110); 5.4 G (Sophono without abutment); and 1.1 G (Cochlear Baha 5). The strength of the magnetic field decreased exponentially with distance from the device, as illustrated in Figs. 8 and 9.

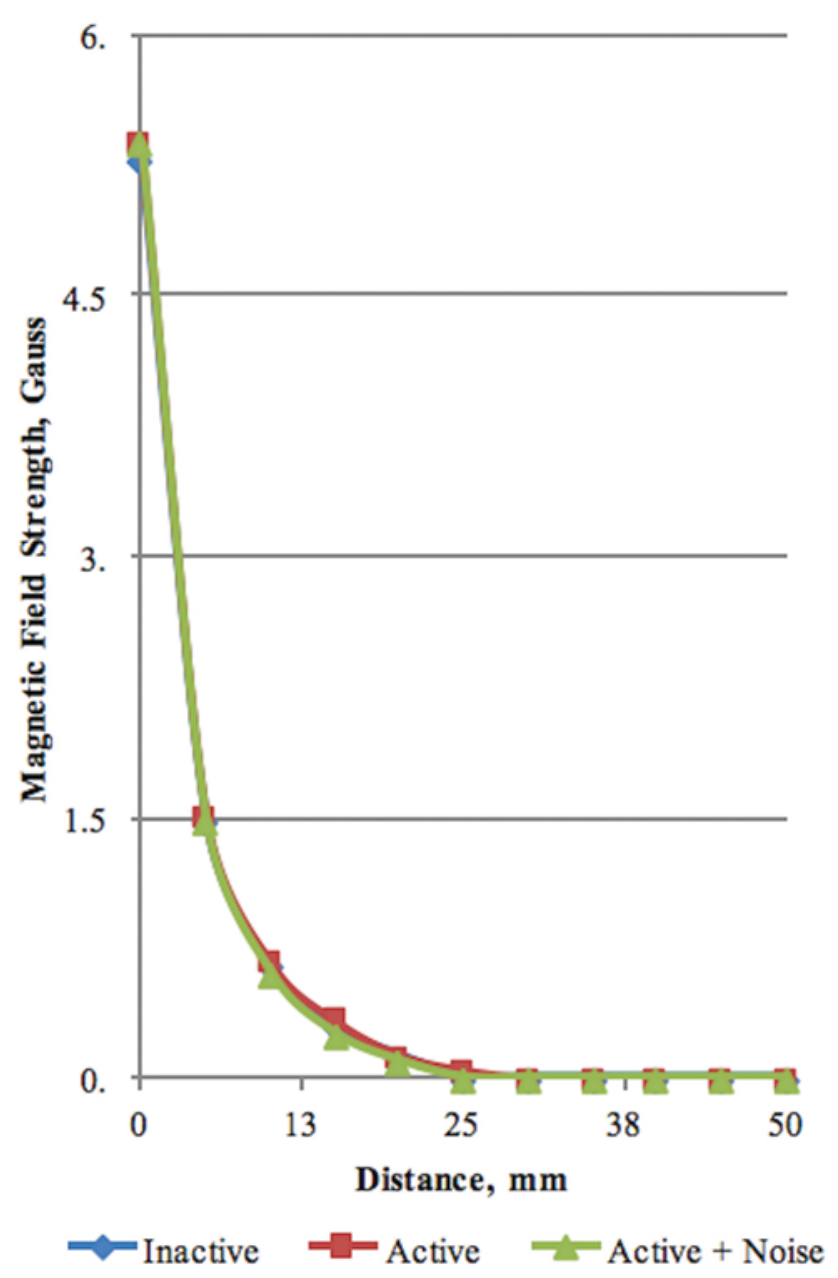

FIG. 5. Graph of magnetic field strength as a function of distance from the Sophono without attachment. Figure is available in color online only.

\section{Reprogramming Events}

\section{Distance Modeling}

No shunt valves were reprogrammed by the Cochlear Baha 5, Cochlear BP110, or Oticon Ponto Plus Power devices at any distance increment. Additionally, none of the shunt valves were reprogrammed following rotation of the valve while in direct contact with the Baha 5, BP110, or Oticon OIHDs. However, the Codman Certas, Codman Hakim, and Medtronic Strata II valves were reprogrammed when rotated in contact with the Sophono abutment.

\section{Anatomical Modeling}

The shunt valves were not inadvertently reprogrammed at any position in the anatomical model, regardless of the OIHD-valve pair tested. The Sophono magnet did not reprogram any valve at any anatomical position.

\section{Discussion}

The maximum magnetic field strength generated by the Cochlear Baha 5, Cochlear BP110, and Oticon Ponto Plus Power OIHDs was low and declined rapidly with distance from the device. The maximum magnetic field strength emitted was below the level of $80 \mathrm{G}$ recommended as 

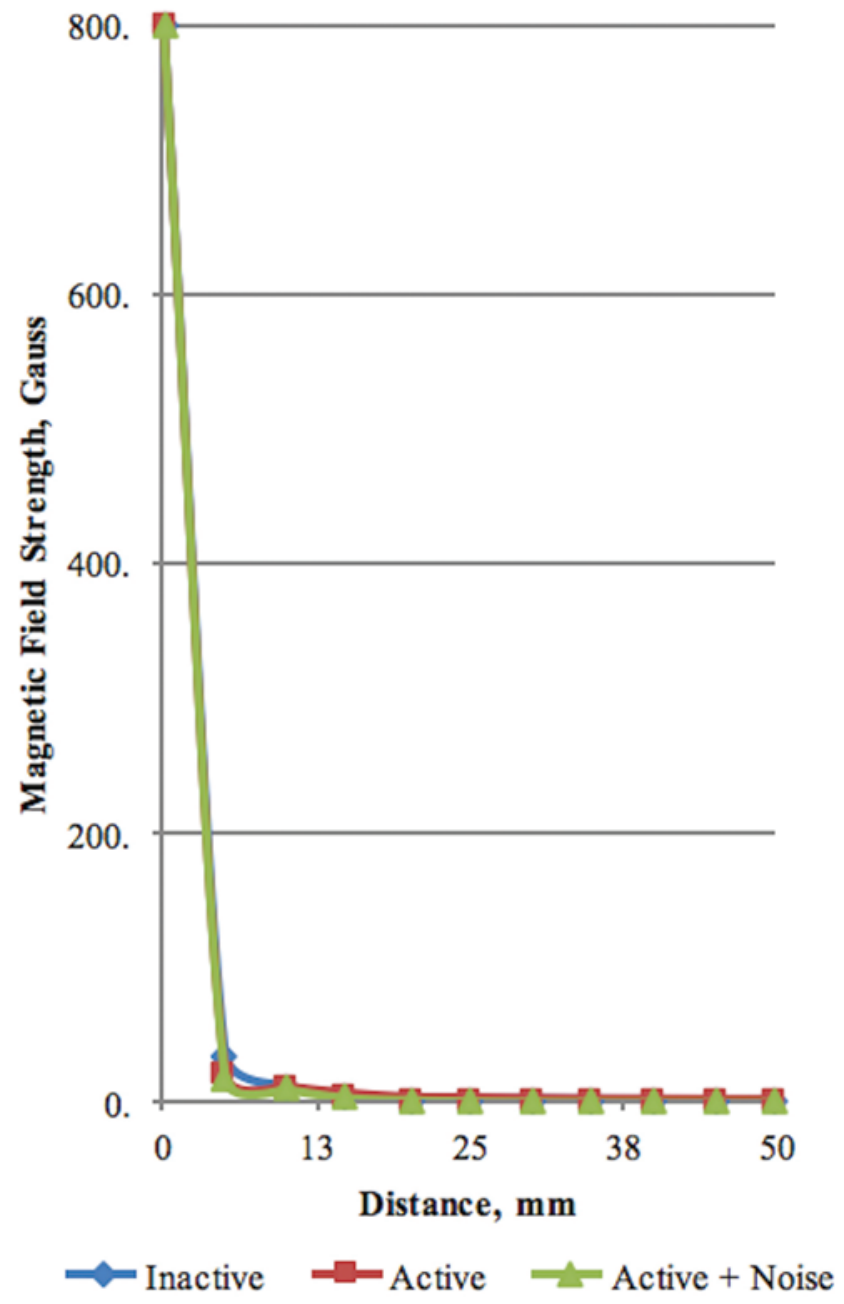

FIG. 6. Graph of magnetic field strength as a function of distance from the Sophono with attachment. Figure is available in color online only.

the safe field limit by valve manufacturers. The OIHDs in this study were chosen based on being the most commonly encountered devices on the market in our experi-
TABLE 1. The mean difference in magnetic field in the 3 states tested

\begin{tabular}{|c|c|c|c|c|c|}
\hline \multirow[b]{2}{*}{ Device \& State } & \multicolumn{5}{|c|}{ Magnetic Field Strength (G) } \\
\hline & $\begin{array}{c}\text { Baha } \\
5\end{array}$ & BP110 & $\begin{array}{l}\text { Plus } \\
\text { Power }\end{array}$ & Sophono & $\begin{array}{c}\text { Sophono } \\
\text { w/ } \\
\text { Abutment }\end{array}$ \\
\hline \multicolumn{6}{|l|}{ Off (inactive) } \\
\hline \multicolumn{6}{|l|}{ Baha 5} \\
\hline BP110 & $35.1^{*}$ & & & & \\
\hline Plus Power & $47.6^{*}$ & $-12.5^{*}$ & & & \\
\hline Sophono & 4.2 & $30.9^{*}$ & $43.4^{*}$ & & \\
\hline $\begin{array}{l}\text { Sophono w/ } \\
\text { abutment }\end{array}$ & $798.9^{*}$ & $763.8^{*}$ & $751.3^{*}$ & $794.7^{*}$ & \\
\hline \multicolumn{6}{|l|}{ On (active) } \\
\hline \multicolumn{6}{|l|}{ Baha 5} \\
\hline BP110 & $35.9^{*}$ & & & & \\
\hline Plus Power & $1.96^{*}$ & $-33.9^{*}$ & & & \\
\hline Sophono & $4^{*}$ & $-31.9^{*}$ & $2.1^{*}$ & & \\
\hline $\begin{array}{l}\text { Sophono w/ } \\
\text { abutment }\end{array}$ & $798.6^{*}$ & $762.8^{*}$ & $796.7^{*}$ & $794.6^{*}$ & \\
\hline \multicolumn{6}{|l|}{$\begin{array}{l}60 \mathrm{~dB} \text { (active in } \\
\text { noise) }\end{array}$} \\
\hline \multicolumn{6}{|l|}{ Baha 5} \\
\hline BP110 & $36.2^{*}$ & & & & \\
\hline Plus Power & $6.2^{*}$ & $-30^{*}$ & & & \\
\hline Sophono & $4.3^{*}$ & $-31.9^{*}$ & $-1.9^{*}$ & & \\
\hline $\begin{array}{r}\text { Sophono w/ } \\
\text { abutment }\end{array}$ & $798.9^{*}$ & $762.7^{*}$ & $792.7^{*}$ & $794.6^{*}$ & \\
\hline
\end{tabular}

ence. There was no difference in emitted magnetic field with the devices inactive, active, and active in noise for any device except for the Oticon Ponto Plus Power OIHD. As shown in Fig. 7, there was a statistically significant difference between the off, on, and on with noise states for this device. Nevertheless, all values in these states for this device were $<80 \mathrm{G}$. The only field measurement $>80 \mathrm{G}$

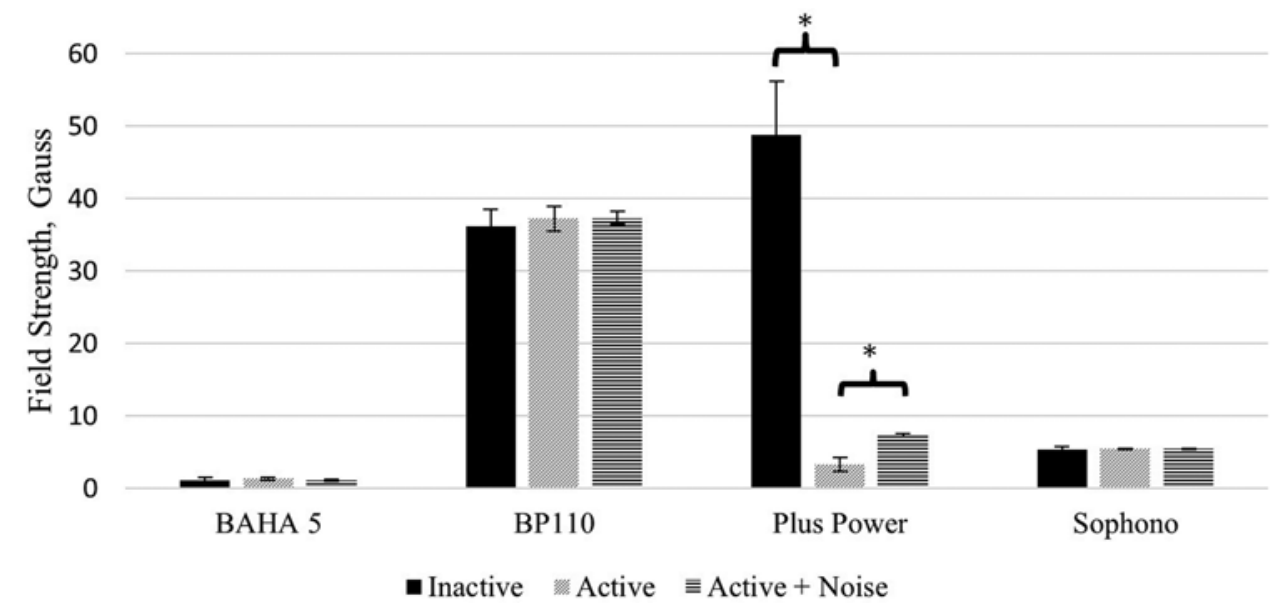

FIG. 7. Bar graph showing a comparison of the maximum magnetic field generated by each device in the inactive, active, and active in background noise states. Error bars denote the SD; ${ }^{*}$ significant at $p<0.001$. 


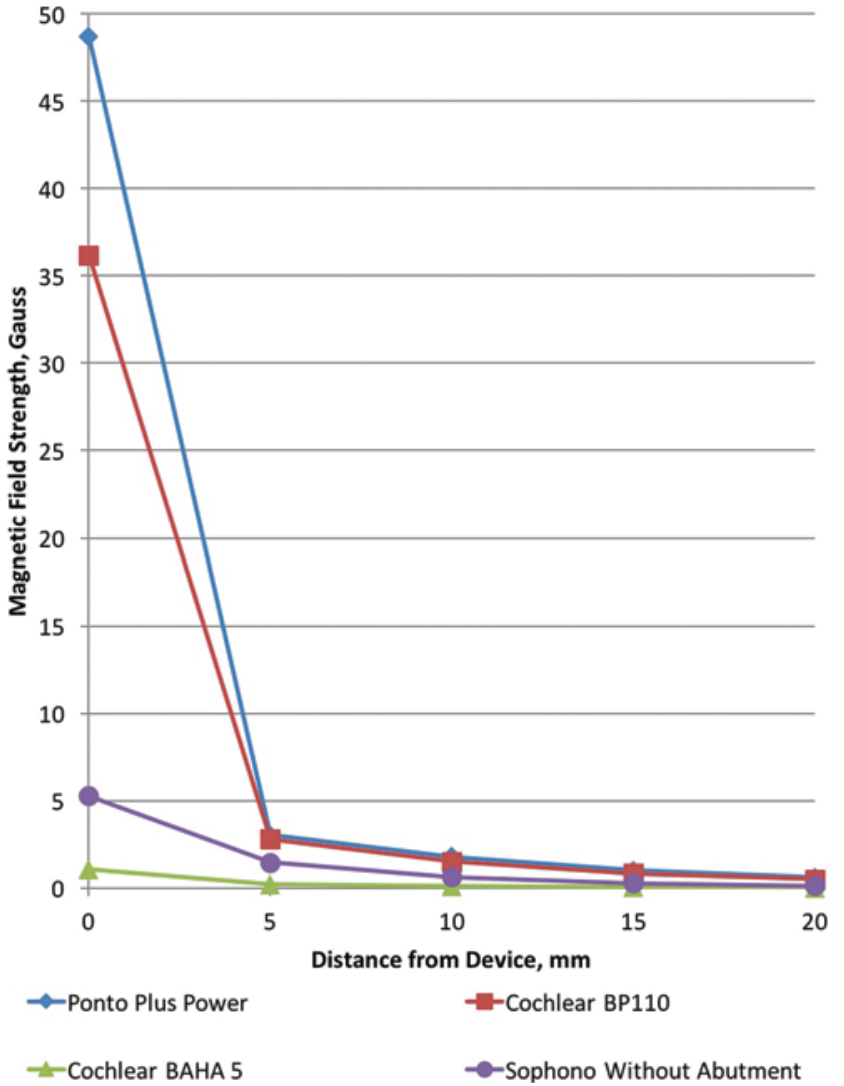

FIG. 8. Graph showing maximum magnetic field strength for each OIHD as a function of distance. Figure is available in color online only.

was for the Sophono abutment, which was outside the limits of the Gaussmeter at $>800 \mathrm{G}$. The magnet strength for the Sophono decreased rapidly as a function of distance, to safe levels at $5 \mathrm{~mm}$. The exponential decline in magnet field strength is consistent with the established formula for magnet strength declining as a function of distance cubed.

We found no scenario in the distance or anatomical models in which programmable shunt valve settings with the Cochlear Baha 5, Cochlear BP110, or Oticon Ponto Plus Power devices were altered. This includes anatomical trials assessing direct contact with the rotational component as well as the ipsilateral parietooccipital valve location. However, rotating the Codman Hakim, Codman Certas, and Medtronic Strata II valves in direct contact with Sophono abutment induced reprogramming. The decline in magnet field strength with distance and the overlying skin thickness encountered when using this device in vivo may reduce the risk of shunt reprogramming. Anatomical modeling revealed no change in valve setting for any valve location with the Sophono device or its abutment. We recognize that there may be variations in valve and bur hole location for shunt insertion that differ from our anatomical studies, but given the minimal interactions observed at even very close distances, this is not thought to be significant. Therefore, the scenario that caused erroneous valve adjustment may be unlikely to occur during actual use of the device. An artificial tissue layer was not used in this study, but it stands to reason that such a layer would fur-

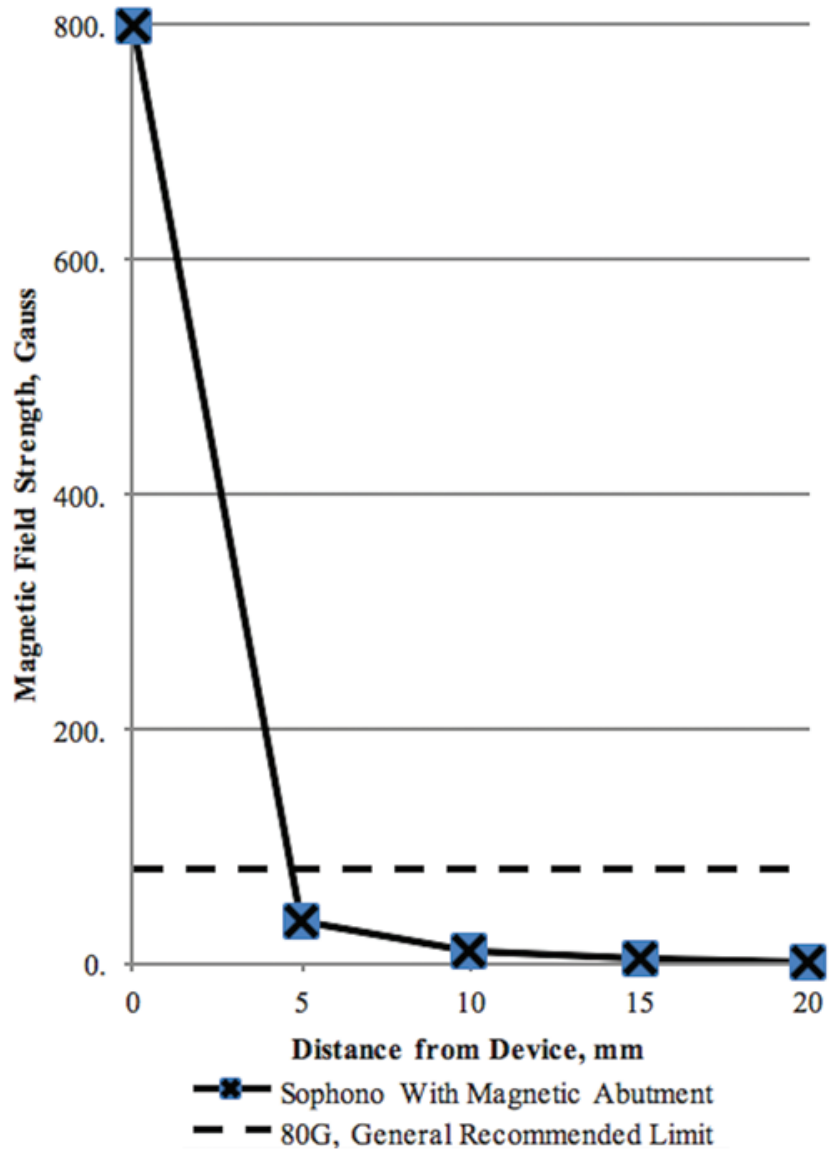

FIG. 9. Graph showing maximum magnetic field strength for the Sophono device with its abutment as a function of distance. Figure is available in color online only.

ther increase the distance between the devices and therefore reduce the already minimal interactions.

There is currently a limited amount of literature regarding the interaction between hearing devices and programmable shunt valves. In a case described by Wiet and El-Kashlan, a 7-month-old infant underwent removal of a programmable shunt valve and replacement with a nonprogrammable valve prior to insertion of bilateral CIs. In their paper, the authors also measure magnetic field strength as a function of distance from the CI magnets. Consistent with our experimental findings, they identified an exponential loss in field strength with increasing distance from the source. ${ }^{10}$ Based on their findings, they recommend the use of a nonprogrammable valve or placement of the valve in a location other than the head if CI insertion is anticipated. An et al. described a case of ipsilateral insertion of a CI in a patient with a frontally located shunt with a programmable valve. In their case, the CI did not affect the setting of the programmable valve. Their device was approximately $8 \mathrm{~cm}$ away from the shunt valve. They recommend a minimum distance of $2 \mathrm{~cm}$ between the CI and a programmable shunt valve to avoid erroneous adjustment. ${ }^{1}$ In a review by Chadwick et al., considerations for pre-, intra-, and postoperative management of patients with both CIs and VP shunts were discussed. ${ }^{3}$ They mentioned that the goal for magnetic field strength should be $<80 \mathrm{G}$. 
To our knowledge, this is the first investigation of the interaction between OIHDs and programmable shunt valves. Limitations of the study include an in vitro model, which may have different characteristics than living tissue. This effect was minimized through use of anatomical modeling on a 3D printed skull. Magnetic interference was minimized by zeroing the device prior to each measurement trial.

\section{Conclusions}

This is the first study evaluating the interaction between OIHDs and programmable VP shunt valves. Our findings suggest that it is safe to use these devices together without changing to a nonprogrammable valve or moving the shunt valve to a more distant location. However, if the Medtronic Sophono device is used, care should be taken to ensure that the valve is at least $\geq 5 \mathrm{~mm}$ away from the shunt valve.

\section{References}

1. An YH, Song SJ, Yoon SW, Kim JH, Shim HJ: Cochlear implantation for total deafness after ipsilateral ventriculoperitoneal shunt surgery: technical report. Acta Neurochir (Wien) 153:2479-2483, 2011

2. Anderson RC, Walker ML, Viner JM, Kestle JR: Adjustment and malfunction of a programmable valve after exposure to toy magnets. Case report. J Neurosurg 101 (2 Suppl):222-225, 2004

3. Chadwick KA, Moore J, Tye GW, Coelho DH: Management of patients with cochlear implants and ventriculoperitoneal shunts. Cochlear Implants Int 15:185-190, 2014

4. Lee L, King NK, Kumar D, Ng YP, Rao J, Ng H, et al: Use of programmable versus nonprogrammable shunts in the management of hydrocephalus secondary to aneurysmal subarachnoid hemorrhage: a retrospective study with cost-benefit analysis. J Neurosurg 121:899-903, 2014

5. Nomura S, Fujisawa H, Suzuki M: Effect of cell phone magnetic fields on adjustable cerebrospinal fluid shunt valves. Surg Neurol 63:467-468, 2005

6. Spader HS, Ratanaprasatporn L, Morrison JF, Grossberg JA, Cosgrove GR: Programmable shunts and headphones: Are they safe together? J Neurosurg Pediatr 16:402-405, 2015
7. Strahle J, Collins K, Stetler WR Jr, Smith BW, Garton T, Garton C, et al: Effect of amusement park rides on programmable shunt valve settings. Pediatr Neurosurg 49:21-23, 2013

8. Strahle J, Selzer BJ, Muraszko KM, Garton HJ, Maher CO: Programmable shunt valve affected by exposure to a tablet computer. J Neurosurg Pediatr 10:118-120, 2012

9. Utsuki S, Shimizu S, Oka H, Suzuki S, Fujii K: Alteration of the pressure setting of a Codman-Hakim programmable valve by a television. Neurol Med Chir (Tokyo) 46:405407, 2006

10. Wiet RM, El-Kashlan HK: Cochlear implantation in the presence of a programmable ventriculoperitoneal shunt. Otol Neurotol 30:704-707, 2009

11. Zuzak TJ, Balmer B, Schmidig D, Boltshauser E, Grotzer MA: Magnetic toys: forbidden for pediatric patients with certain programmable shunt valves? Childs Nerv Syst 25:161-164, 2009

\section{Disclosures}

Dr. Albers received clinical or research support for the study described from Teva Pharmaceuticals. The authors gratefully acknowledge the following companies for their material support for the purpose of the study: Aesculap, Inc. (Center Valley, PA); Cochlear Ltd. (Sydney, Australia); Codman Neuro (Raynham, MA); Medtronic (Dublin, Republic of Ireland); and Oticon (Somerset, $\mathrm{NJ}$ ).

\section{Author Contributions}

Conception and design: Elbabaa, Costa. Acquisition of data: Pierson, Wehrmann, Albers, El Tecle. Analysis and interpretation of data: Elbabaa, Pierson, Wehrmann, Albers, El Tecle. Drafting the article: Elbabaa, Pierson, Wehrmann, Albers. Critically revising the article: all authors. Reviewed submitted version of manuscript: Elbabaa, Pierson, Albers, Costa. Approved the final version of the manuscript on behalf of all authors: Elbabaa. Statistical analysis: Albers. Administrative/technical/material support: Elbabaa, Costa. Study supervision: Elbabaa, Costa.

\section{Correspondence}

Samer K. Elbabaa, Division of Pediatric Neurosurgery, Saint Louis School of Medicine, 1465 S Grand Blvd., St. Louis, MO 63104.email: selbabaa@slu.edu. 